\title{
MULTIPLIERS FOR EIGENFUNCTION EXPANSIONS OF SOME SCHRÖDINGER OPERATORS
}

\author{
KRZYSZTOF STEMPAK
}

\begin{abstract}
Let $G$ be a graded nilpotent Lie group and let $L$ be a positive Rockland operator on $G$. Let $E_{\lambda}$ denote the spectral resolution of $L$ on $L^{2}(G)$. A sufficient condition is given under which a function $m$ on $\mathbf{R}^{+}$is a $L^{p}$-multiplier for $L$, $1<p<\infty$; that is $\left\|\int_{0}^{\infty} m(\lambda) d E_{\lambda} f\right\|_{p} \leqslant C_{p}\|f\|_{p}$ for a constant $C_{p}, f \in L^{p}(G) \cap$ $L^{2}(G)$. Then the same is done for an operator $\pi(L)$, where $\pi$ is a unitary representation of $G$ induced from a unitary character of a normal connected subgroup $H$ of $G$. Hence the case of the Hermite operator $-d^{2} / d x^{2}+x^{2}$ is covered and an $L^{p}$-multiplier theorem for classical Hermite expansions is obtained.
\end{abstract}

1. Let $L$ be an essentially selfadjoint on its domain, positive, densely defined operator on $L^{2}(X)$, where $X$ is a measure space. Let $E_{\lambda}$ be a spectral resolution of the identity for which

$$
L f=\int_{0}^{\infty} \lambda d E_{\lambda} f, \quad f \in \operatorname{Dom}(L) .
$$

If $m$ is a bounded measurable function on $\mathbf{R}^{+}$, we write $m(L)$ for the "multiplier operator"

$$
m(L) f=\int_{0}^{\infty} m(\lambda) d E_{\lambda} f, \quad f \in L^{2}(X),
$$

which is bounded on $L^{2}(X)$.

The question about conditions on $m$ which guarantee that $m(L)$ is bounded on $L^{p}(X)$ has been raised by E. M. Stein [9] and discussed in a number of papers afterwards. Thus we say that a function $m$ is an $L^{p}$-multiplier for the operator $L$ if there exists a constant $C_{p}$ such that for all $f$ in $L^{p}(X) \cap L^{2}(X)$

$$
\|m(L) f\|_{p} \leqslant C_{p}\|f\|_{p}
$$

In [1] A. Bonami and J. L. Clerc present a technique, based on investigation of some $g$-functions of Paley-Littlewood type, which allows them to obtain an $L^{p}$-multiplier theorem in the case when $L$ is the Laplace-Beltrami operator on a compact, riemannian manifold. An estimate due to L. Hörmander (cf. [1, p. 259]) plays here the crucial role.

Received by the editors February 14, 1984.

1980 Mathematics Subject Classification. Primary 43A80, 22E30; Secondary 33A75.

Key words and phrases. Multiplier, eigenfunction expansion, Rockland operator, Hermite function, homogenous type space, maximal function, Riesz means. 
Recently, A. Hulanicki and J. W. Jenkins $[6,7,8]$ have obtained similar estimates for a class of operators which are of different types and include, among others, operators $-\Delta+V$, where $\Delta$ is the Laplacian on $R^{k}$ and $V$ is a sum of squares of polynomials.

In this paper we observe that a method used by Bonami and Clerc can be applied to results of Hulanicki and Jenkins.

We obtain a stronger version of the Hulanicki-Stein multiplier theorem for a sub-Laplacian (cf. [5]) and for a positive Rockland operator (cf. [6]), and we produce new multiplier theorems for the eigenfunction expansions of Schrödinger type operators.

We illustrate our results by the following theorem concerning the Hermite expansion.

THEOREM 1. There exists an integer $N$ such that if $m \in C^{N}\left(\mathbf{R}^{+}\right)$satisfies the conditions

$$
|m(\lambda)| \leqslant M, \quad \sup _{A>0} \frac{1}{A} \int_{0}^{A} \lambda^{k}\left|m^{(k)}(\lambda)\right| d \lambda \leqslant M, \quad k=1, \ldots, N,
$$

then for every $1<p<\infty$ the sequence $m(2 j+1), j=0,1, \ldots$, is a multiplier on $L^{p}(\mathbf{R})$ with respect to the Hermite expansion; that is, for all $f$ in $L^{p}(\mathbf{R}) \cap L^{2}(\mathbf{R})$

$$
\left\|\sum m(2 j+1)\left\langle h_{j}, f\right\rangle h_{j}\right\|_{p} \leqslant C_{p}\|f\|_{p}
$$

with a constant $C_{p}$.

Here $h_{j}$ is a jth Hermite function and

$$
\left\langle h_{j}, f\right\rangle=\int_{\mathbf{R}} h_{j}(x) f(x) d x .
$$

2. Let $E_{\lambda}$ be a positive (i.e. $E_{\lambda}=0$ for $\lambda \leqslant 0$ ) spectral resolution of the identity on $L^{2}(X)$. Denote by $S_{R}^{\delta}, R>0$, the $\delta$-Riesz mean

$$
S_{R}^{\delta} f=\int_{0}^{R}\left(1-\frac{\lambda}{R}\right)^{\delta} d E_{\lambda} f, \quad f \in L^{2}(X) .
$$

We suppose that $E_{\lambda}$ has the following properties:

(a) For $p>1$ and a positive integer $\delta$ the Marcinkiewicz-Zygmund property holds, i.e.

there exists a constant $B_{p}$ such that for an arbitrary sequence of positive numbers $R_{j}$ and for an arbitrary sequence of functions $f_{j} \in L^{p}(X) \cap L^{2}(X)$ we have

$$
\left\|\left(\sum\left|S_{R_{j}}^{\delta} f_{j}\right|^{2}\right)^{1 / 2}\right\|_{p} \leqslant B_{p}\left\|\left(\sum\left|f_{j}\right|^{2}\right)^{1 / 2}\right\|_{p}
$$

(b) The operators $T^{t}=\int_{0}^{\infty} \exp (-\lambda t) d E_{\lambda}$ are contractions on all $L^{p}(X), 1 \leqslant p \leqslant$ $\infty$. 
The following theorem is essentially due to Bonami and Clerc (cf. [1, p. 260]; the details of the proof extracted from [1] and adapted to our situation can be found in [10]).

THEOREM A. Let $E_{\lambda}$ be a positive spectral resolution of the identity which satisifies (a) and (b). If a function $m \in C^{\delta+1}\left(\mathbf{R}^{+}\right)$satisfies the conditions

$$
|m(\lambda)| \leqslant M, \quad \sup _{A>0} \frac{1}{A} \int_{0}^{A} \lambda^{j}\left|m^{(j)}(\lambda)\right| d \lambda \leqslant M, \quad j=1, \ldots, \delta+1,
$$

then $m$ is an $L^{p}$-multiplier for $E_{\lambda}$ : that is, there exists a constant $C_{p}$ such that

$$
\left\|\int_{0}^{\infty} m(\lambda) d E_{\lambda} f\right\|_{p} \leqslant C_{p}\|f\|_{p}, \quad f \in L^{p}(X) \cap L^{2}(X) .
$$

The constant $C_{p}$ does not depend on the function $m$ but only on $M$.

In the sequel we deal with spaces of homogeneous type, in the sense of [2] only.

Let us recall that a topological space $X$ equipped with a continuous pseudometric $\rho$ and with a measure $\mu$, which, for a constant $C$, satisfies

$$
\mu\left(B_{r}(y)\right) \leqslant C \mu\left(B_{r / 2}(x)\right), \quad x, y \in X, r>0,
$$

is a space of homogeneous type and the Hardy-Littlewood maximal function

$$
m^{*} f(x)=\sup _{r>0} \mu\left(B_{r}(x)\right)^{-1} \int_{B_{r}(x)}|f(y)| d \mu(y)
$$

is of weak type $(1,1)$ (cf. [2]). Here $B_{r}(x)$ denotes the ball $B_{r}(x)=\{y: \rho(x, y)<r\}$.

Moreover, the following Marcinkiewicz-Zygmund type inequality due to $C$. Fefferman and E. M. Stein is valid (cf. [4]; see also [1]).

THEOREM B. Let $\mu$ be a measure and $\rho$ a pseudometric on $X$ such that (2.2) holds. Then for every $1<p<\infty$ there exists a constant $D_{p}$, such that for an arbitrary sequence of functions $f_{i}$ we have

$$
\left\|\left(\sum\left|m^{*} f_{i}\right|^{2}\right)^{1 / 2}\right\|_{p} \leqslant D_{p}\left\|\left(\sum\left|f_{i}\right|^{2}\right)^{1 / 2}\right\|_{p} .
$$

Observe that if the spectral measure $E_{\lambda}$ on $L^{2}(X)$ satisfies the estimate

$$
\sup _{R>0}\left|S_{R}^{\delta} f\right| \leqslant C m^{*} f
$$

then, in virture of Theorem $\mathrm{B}, E_{\lambda}$ has for every $p, 1<p<\infty$, the MarcinkiewiczZygmund property (2.1).

3. Let $G$ be a graded nilpotent Lie group of [5]. $G$ endowed with the Haar measure and the pseudometric generated by the homogeneous norm is of homogeneous type (cf. [5]).

Let $L$ be a positive Rockland operator on $G$ that is a homogeneous, left-invariant differential operator such that $\pi(L)$ is injective on $C^{\infty}$-vectors for every irreducible, nontrivial unitary representation $\pi$ of $G$. It is known (cf. [5]) that $L$ is hypoelliptic, and as such it is essentially selfadjoint on $L^{2}(G)$. 
Let $E_{L}(\lambda)$ be a spectral resolution of the identity for $L$. The operators $T^{t}=$ $\int_{0}^{\infty} \exp (-\lambda t) d E_{L}(\lambda)$ are contractions on all $L^{p}(G), 1 \leqslant p \leqslant \infty$ (cf. [5]).

As we have mentioned before, Hulanicki and Jenkins have shown (cf. [6, 8]; see also [7]) that there exists an integer $N(L)$ such that

$$
\sup _{R>0}\left|S_{R}^{N(L)} f\right| \leqslant C m_{G}^{*} f,
$$

where $m_{G}^{*}$ is the Hardy-Littlewood maximal function on $G$ and $S_{R}^{N(L)}, R>0$, are Riesz means corresponding to $E_{L}(\lambda)$.

Thus we can state the following

Proposition 1. Let $L$ be a positive Rockland operator and let $E_{L}(\lambda)$ be a spectral resolution of $L$. Let $N(L)$ be an integer such that (3.1) holds. If $m \in C^{N(L)+1}\left(\mathbf{R}^{+}\right)$ satisfies the conditions

$$
|m(\lambda)| \leqslant M, \quad \sup _{A>0} \frac{1}{A} \int_{0}^{A} \lambda^{k}\left|m^{(k)}(\lambda)\right| d \lambda \leqslant M, \quad k=1, \ldots, N(L)+1,
$$

for a constant $M$, then for every $p, 1<p<\infty$, the function $m$ is an $L^{p}$-multiplier for the operator $L$.

4. Let $G$ be an arbitrary nilpotent Lie group and let $\pi$ be a representation of $G$ induced from a unitary character of a normal connected subgroup $H$ of $G$. The operators $\pi(x), x \in G$, act on $L^{2}(G / H)$.

Let $X_{1}, \ldots, X_{k}$ be elements which generate the Lie algebra of $G$. Put

$$
L=\sum_{j=1}^{k}(-1)^{n_{j}} X_{j}^{2 n_{j}}
$$

where $n_{j}, j=1, \ldots, k$, are arbitrary positive integers. Then $\pi(L)$ is a positive essentially selfadjoint operator on $L^{2}(G / H)$. Denote by $E_{\pi(L)}(\lambda)$ the spectral resolution of $\pi(L)$.

Proposition 2. Let $L$ and $E_{\pi(L)}$ be as above. Then there exists an integer $N$ such that if $m \in C^{N}\left(\mathbf{R}^{+}\right)$satisfies the conditions

$$
|m(\lambda)| \leqslant M, \quad \sup _{A>0} \frac{1}{A} \int_{0}^{A} \lambda^{k}\left|m^{(k)}(\lambda)\right| d \lambda \leqslant M, \quad k=1, \ldots, N,
$$

for a constant $M$, then for every $p, 1<p<\infty$, the function $m$ is an $L^{p}$-multiplier for the operator $\pi(L)$.

Proof. Let us repeat, for the reader's convenience, the same arguments as in the proof of Theorem 2.6 of [8] which allows us to assume that $G$ is stratified and that $L$ is a Rockland operator. Let $\underline{G}$ be the nilpotent free group of the same nilpotency class as $G$ and let $\underline{X}_{1}, \ldots, \underline{X}_{k}$ be the free generators of the Lie algebra of $\underline{G}$. Denote by $\alpha$ the homomorphism of $\underline{G}$ onto $G$ sending $\exp \underline{X}_{j}$ onto $\exp X_{j}$. Thus $\pi^{\prime}=\pi \circ \alpha$ is a representation of $\underline{G}$ induced by a unitary character of the normal connected subgroup $\underline{H}=\alpha^{-1}(H)$ of $\underline{G}$. Define the dilations $\delta_{t}, t>0$, of free Lie algebras of $\underline{G}$ by putting

$$
\delta_{t} \underline{X}_{-j}=t^{1 / 2 n_{j}} \underline{X}_{j}, \quad 1, \ldots, k
$$


Then

$$
\underline{L}=\sum_{j=1}^{k}(-1)^{n_{j}} \underline{X}_{j}^{2 n_{j}}
$$

is a Rockland operator on $\underline{G}, \delta_{t} \underline{L}=t L$, and $\pi^{\prime}(\underline{L})=\pi(L)$.

Proposition 2.2 of [8] asserts that $G / H$, equipped with the Haar measure and the pseudometric $\rho$ defined by

$$
\rho(x, y)=\inf \left\{\left|x y^{-1} z\right|: z \in H\right\},
$$

is a space of homogenous type. Denote by $m_{G / H}^{*}$ the corresponding Hardy-Littlewood maximal function.

Consequently, Theorem 2.6 of [8] applied to the function $K(\lambda)=(1-\lambda)^{N}$ for $\lambda \leqslant 1$ and $K(\lambda)=0$ for $\lambda>1$, for sufficiently large $N$, allows us to obtain an estimate

$$
\sup _{R>0}\left|\int_{0}^{R}\left(1-\frac{\lambda}{R}\right)^{N} d E_{\pi(L)}(\lambda) f\right| \leqslant C \cdot m_{G / H}^{*}(f) .
$$

Now, it remains to note that $\pi(L)$ generates a semigroup of contractions on all $L^{p}(G / H), 1 \leqslant p \leqslant \infty$. Denote by $E_{L}(\lambda)$ the spectral resolution of $L$ in $L^{2}(G)$. By [5]

$$
\int_{0}^{\infty} \exp (-\lambda t) d E_{L}(\lambda) f=k_{t^{*}} f, \quad f \in L^{2}(G),
$$

where $k_{t} \in L^{1}(G),\left\|k_{t}\right\|_{1}=1$. Note that $\left\{\pi\left(k_{t}\right)\right\}_{t>0}$ is a semigroup on $L^{2}(G / H)$ and that, for $f \in L^{2}(G / H)$ and $\varphi \in C_{c}(G)$,

$$
\lim _{t \rightarrow 0} t^{-1}\left\{\pi\left(k_{t}\right) \pi(\varphi) f-\pi(\varphi) f\right\}=\pi(L \varphi) f=\pi(L) \pi(\varphi) f .
$$

Thus, $\pi(L)$ is the infinitesimal generator for $\left\{\pi\left(k_{t}\right)\right\}_{t>0}$, which gives

$$
\int_{0}^{\infty} \exp (-\lambda t) d E_{\pi(L)}(\lambda) f=\pi\left(k_{t}\right) f, \quad f \in L^{2}(G / H) .
$$

The operators $\pi(x)$ act on $L^{2}(G / H)$ by

$$
\pi(x) f(\dot{y})=a(x, \dot{y}) f(\dot{y} \dot{x}),
$$

where the scalar function $a$ is such that $|a(x, y)|=1$. Thus $\pi(x)$ is a contraction on every $L^{p}(G / H)$ and so the same is true for the operator

$$
\pi\left(k_{t}\right)=\int_{G} \pi(x) k_{t}(x) d x .
$$

This completes the proof of the proposition.

Now, to see that Theorem 1 is a consequence of Proposition 2 we note that the Hermite operator $-d^{2} / d x^{2}+x^{2}$ is of the form $\pi(L)$, where $L$ is the sub-Laplacian on the Heisenberg group, and $\pi$ is the Schrödinger representation on it. 
Finally we mention also that in virtue of the recent results of W. Cupala [3], following the ideas of Hulanicki and Jenkins [8], our Proposition 2 produces an $L^{p}$-multiplier theorem for the eigenfunction expansions of the operators of the form

$$
(-1)^{k} d^{2 k} / d x^{2 k}+p(x)
$$

where $p(x)$ is a positive polynomial and $k$ is an arbitrary positive integer.

ACKNOWLEDGEMENTS. I would like to thank Andrzej Hulanicki for valuable suggestions.

\section{REFERENCES}

1. A. Bonami and J. L. Clerc, Sommes de Cesàro et multiplicateurs des développements en harmoniques sphériques, Trans. Amer. Math. Soc. 183 (1973), 223-263.

2. R. Coifman and G. Weiss, Analyse harmonique non-commutative sur certains espaces homogènes, Lecture Notes in Math., Vol. 242, Springer-Verlag, Berlin and New York, 1971.

3. W. Cupala, Certain Schrödinger operators as images of sublaplacians on nilpotent Lie groups, Studia Math. (to appear).

4. C. Fefferman and E. M. Stein, Some maximal inequalities, Amer. J. Math. 93 (1971), 107-115.

.5. G. Folland and E. M. Stein, Hardy spaces on homogenous groups, Princeton Univ. Press, Princeton, N. J., 1982.

6. A. Hulanicki, A functional calculus for Rockland operators on nilpotent Lie groups, Studia Math. 78 (to appear).

7. A. Hulanicki and J. W. Jenkins, Almost everywhere summability on nilmanifolds, Trans. Amer. Math. Soc. 278 (1983), 703-715.

8. Nilpotent Lie groups and summability of eigen-function expansions of Schrödinger operators, Studia Math. (to appear).

9. E. M. Stein, Topics in harmonic analysis related to the Littlewood-Paley theory, Princeton Univ. Press, Princeton, N. J., 1970.

10. K. Stempak, Multipliers for eigenfunction expansions of some Schrödinger operators, 1984.

Institute of Mathematics, University of Wrockaw, Pl. Grunwaldzki 2 4, PL 50 - 384 Wrocraw, Poland 